\title{
Obstetric intensive care unit admission: a six year cohort study
}

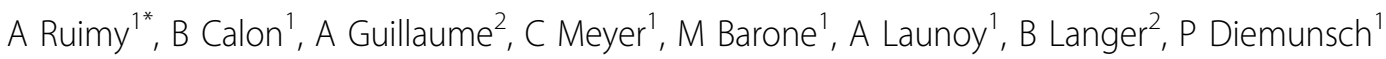 \\ From ESICM LIVES 2015 \\ Berlin, Germany. 3-7 October 2015
}

\section{Introduction}

One in a thousand pregnancies will be complicated by serious maternal morbidity warranting intensive care unit (ICU) admission before, during or after delivery. Between 2007 and 2009 maternal mortality in France was one in ten thousand, about half of them are considered preventable.

\section{Objectives}

In order to assess and improve avoidable severe maternal morbidity (SMM), whether lethal or not, a prospective analysis was undertaken in a tertiary care obstetric ICU (TCO-ICU) over a six year period.

\section{Methods}

Between January 2007 and December 2012 data were collected on all obstetric and post-partum admissions in the Strasbourg (France) TCO-ICU.

\section{Results}

Among the 305 TCO-ICU admissions, 2 patients presented with avoidable (iatrogenic) non-lethal morbidity and 8 patients died, all of them from non-avoidable causes. No deaths occurred in the 2 main groups, hypertensive disorders of pregnancy and major obstetric hemorrhage.

\section{Conclusions}

Optimal cooperation between our tertiary facilities and non-teaching hospitals and clinics as well as the prehospital transport systems allows significant limitations in avoidable obstetric maternal morbidity and mortality. Cerebral hemorrhage and amniotic fluid embolism carry the heaviest toll in non-avoidable deaths.
Table 1. lindications for ICU admission.

\begin{tabular}{llll}
\hline $\begin{array}{l}\text { indication for ICU } \\
\text { admission }\end{array}$ & $\begin{array}{l}\text { number of } \\
\text { patients }\end{array}$ & $\%$ & $\begin{array}{l}\text { number of } \\
\text { deaths }\end{array}$ \\
\hline hypertensive disorder & 195 & 64 & 0 \\
\hline Hemorrhage & 51 & 16.7 & 0 \\
\hline infectious disease & 11 & 3.6 & 1 \\
\hline amniotic fluid embolism & 9 & 3 & 2 \\
\hline hepatic disorder & 6 & 2 & 0 \\
\hline cerebral disease & 5 & 1.6 & 3 \\
\hline cardiac disease & 4 & 1.3 & 0 \\
\hline thromboembolism & 3 & 1 & 0 \\
\hline miscellaneous disorders & 16 & 5.4 & 2 \\
\hline
\end{tabular}

\section{Authors' details}

${ }^{1}$ Hôpitaux Universitaires de Strasbourg, Anesthésie Réanimation, Strasbourg, France. ${ }^{2}$ Hôpitaux Universitaires de Strasbourg, Gynécologie Obstétrique, Strasbourg, France.

Published: 1 October 2015

\section{Reference}

1. Zwart JJ, Dupuis JR, Richters A, Ory F, van Roosmalen J: Obstetric intensive care unit admission: a 2-year nationwide population-based cohort study. Intensive Care Med 2010, 36(2):256-263.

doi:10.1186/2197-425X-3-S1-A525

Cite this article as: Ruimy et al:: Obstetric intensive care unit admission: a six year cohort study. Intensive Care Medicine Experimental 2015 3(Suppl 1):A525. 